\title{
miRNA-21 inhibition inhibits osteosarcoma cell proliferation by targeting PTEN and regulating the TGF-ß1 signaling pathway
}

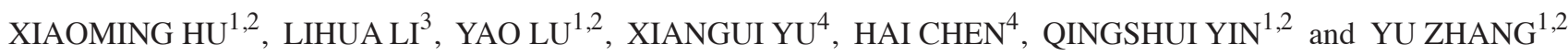 \\ ${ }^{1}$ Department of Orthopedics, Southern Medical University, Guangzhou, Guangdong 510515; \\ ${ }^{2}$ Department of Orthopedics; ${ }^{3}$ Guangdong Key Laboratory of Orthopedic Technology and Implant, \\ Guangzhou General Hospital of Guangzhou Military Command, Guangzhou, Guangdong 510010; \\ ${ }^{4}$ Department of Orthopedics, Anshun City People's Hospital, Anshun, Guizhou 561000, P.R. China
}

Received January 25, 2018; Accepted July 3, 2018

DOI: $10.3892 / \mathrm{ol} .2018 .9177$

\begin{abstract}
The present study aimed to investigate the role of microRNA (miRNA)-21 in the growth of osteosarcoma. A total of 46 patients with osteosarcoma and 20 healthy controls were included in the study. The expression of miRNA-21 was detected by reverse transcription-quantitative polymerase chain reaction in tumor tissues and adjacent healthy tissues from patients with osteosarcoma, as well as the serum of patients with osteosarcoma and the healthy controls was. Receiver operating characteristic curve analysis was performed to evaluate the diagnostic values of serum miRNA-21 for osteosarcoma at different T stages. Survival curves plotted using the Kaplan-Meier method were used to evaluate the prognostic value. miRNA-21 knockdown osteosarcoma cell lines were established and their effects on cell proliferation were explored using a Cell Counting Kit-8 assay. The effect of miRNA-21 knockdown on the protein expression of phosphatase and tensin homolog (PTEN) and transforming growth factor (TGF)- $\beta 1$ was detected by western blot analysis. The expression levels of miRNA-21 in tumor tissues were significantly higher compared with the adjacent healthy tissues in the majority of patients with osteosarcoma. The serum miRNA-21 increased as the T-stage of osteosarcoma increased. Serum miRNA-21 may be used to effectively diagnose osteosarcoma and predict the prognosis of the disease. miRNA-21 knockdown inhibited the proliferation of osteosarcoma and promoted the expression of PTEN and TGF- $\beta 1$ proteins in the osteosarcoma cells. However, TGF- $\beta 1$ inhibitor treatment reduced the inhibitory effects of miRNA-21 knockdown on osteosarcoma cell proliferation. In conclusion, miRNA-21 inhibition may inhibit
\end{abstract}

Correspondence to: Dr Qingshui Yin or Dr Yu Zhang, Department of Orthopedics, Southern Medical University, 1023 South Shatai Road, Baiyun, Guangzhou, Guangdong 510515, P.R. China

E-mail: gz_yqs@126.com

E-mail: luck_2001@126.com

Key words: osteosarcoma, microRNA-21, phosphatase and tensin homolog, transforming growth factor- $\beta 1$, proliferation osteosarcoma cell proliferation by targeting PTEN and regulating the TGF- $\beta 1$ signaling pathway.

\section{Introduction}

Osteosarcoma, or osteogenic sarcoma, is a type of aggressive malignant neoplasm that originates from bone (1). Osteosarcoma is a rare type of malignancy that only accounts for about $1 \%$ of cancers overall, with an incidence of around 5 per 1,000,000 (2). In the United States, osteosarcoma affects about 1,000 new patients every year (3). Osteosarcoma mainly affects young people, and primary conventional osteosarcoma is commonly observed in young patients, while elderly patients are usually affected by secondary osteosarcoma (4). Although various treatment strategies such as chemotherapy, radiation therapy and immunotherapy are efficient for the disease, treatment outcomes are still unsatisfied (5) and the overall 5-years' survival rate is only about $30 \%$ (6). The development of osteosarcoma is a complex process with various factors that involved. Therefore, the in-depth analyses of the pathogenesis of osteosarcoma will definitely improve the treatment of this disease.

MicroRNA, or miRNA, is a group of non-coding RNAs that composed of approximately 22 nucleotides (7). miRNAs are widely distributed in animals, plants and even some viruses to play different roles in almost all critical physiological processes and even pathological changes by its functions of post-transcriptional regulation of gene expression and RNA silencing (8). Oncological studies have also shown that miRNAs are involved in nearly all aspects of the onset, development and progression of different types of malignancies (9). miRNA-21 is usually highly expressed in various types of cancer to play its oncogenic functions (10). However, the involvement of miRNA-21 in osteosarcoma, the mechanism and its prognostic as well as diagnostic values still have not been systemically studied.

\section{Materials and methods}

Patients. A total of 46 patients with osteosarcoma were selected in Guangzhou General Hospital of Guangzhou Military Command from January 2010 to January 2012. Those patients were diagnosed as osteosarcoma by pathological 
and imaging examinations. All patients received surgical resections. Those patients included 26 males and 20 females, and age ranged from 10 to 65 years, with an average age of $32 \pm 9.2$ years. During surgical operations, cancer tissues and adjacent normal tissues within $0.5 \mathrm{~cm}$ around the tumors were collected. Extent of primary tumor was performed according to the criteria established by American Cancer Society. There were 12 cases in T1, 19 cases in T2 and 15 cases in T3 stage. At the same time, 20 healthy controls with similar age and gender distributions were selected to serve as control group. The ethics committee of Guangzhou General Hospital of Guangzhou Military Command approved this study, and all patients signed informed consent. Follow-up study was performed for 6 years to monitor the survival conditions.

Preparation of serum. Fasting blood $(5 \mathrm{ml})$ was extracted from each patient and healthy control on the day of admission. Blood was kept at room temperature for $1.5 \mathrm{~h}$, followed by centrifugation at 2,000 $\mathrm{x}$ g for $20 \mathrm{~min}$ to collect serum. Serum samples were stored at $-80^{\circ} \mathrm{C}$ before RNA extraction.

Cell lines and cell culture. Human osteosarcoma cell lines MG-63 and U2OS were purchased from American Type Culture Collection (ATCC; Manassas, VA, USA). Cells were cultured with Eagle's Minimum Essential Medium (cat. no. 30-2003; ATCC) containing $10 \%$ heat-inactivated fetal bovine serum in an incubator $\left(37^{\circ} \mathrm{C}, 5 \% \mathrm{CO}_{2}\right)$. Cells were harvested during logarithmic growth phase for subsequent experiments.

Establishment of miRNA-21 knockdown cell lines. The pGCMV/EGFP-hsa-miR-21 interference plasmid and control pGCMV/EGFP-hsa-miR-NC plasmid (empty plasmid without the interference) were purchased from GenePharm (Shanghai, China). Plasmid was transfected into cells with a dose of $50 \mathrm{ng} / \mu 1$ using Lipofectamine ${ }^{\circledR} 2000$ Reagent (Invitrogen; Thermo Fisher Scientific, Inc., Waltham, MA, USA).

Cell proliferation assay. Cell Counting Kit-8 (CCK-8; Dojindo Molecular Technologies, Inc., Kumamoto, Japan) was used to monitor cell proliferation according to manufacturer's instructions. Briefly, $100 \mu \mathrm{l}$ of cell suspension containing 3,000 cells was added into each well of 96-well plate. Cells were re-incubated for $12 \mathrm{~h}$, and $10 \mu \mathrm{l}$ of CCK-8 was added 24, 48, 72 and $96 \mathrm{~h}$ later. Absorbance at $450 \mathrm{~nm}$ was measured using Epoch Microplate Spectrophotometer (BioTek Instruments, Inc., Winooski, VT, USA) to calculate cell proliferation rate.

Reverse transcription-quantitative polymerase chain reaction (RT-qPCR). TRIzol reagent (Invitrogen; Thermo Fisher Scientific, Inc., Waltham, MA, USA) was used to extract total RNA from tissues and cells. RNA samples were tested by NanoDrop ${ }^{\mathrm{TM}} 2000$ Spectrophotometers (Thermo Fisher Scientific, Inc.), and only RNA samples with a ratio of A260/A280 between 1.8 and 2.0 were used in reverse transcription to synthesize cDNA using iScript ${ }^{\mathrm{TM}}$ cDNA Synthesis kit (Bio-Rad Laboratories, Inc., Hercules, CA, USA). PCR reactions were performed using $S_{Y B R}{ }^{\circledast}$ Green Real-Time PCR Master Mixes (Thermo Fisher Scientific, Inc.) on the ABI 7500 System. Primers for miRNA-21 were purchased from
Sigma-Aldrich (MIRAP00047; Sigma-Aldrich; Merck KGaA, Darmstadt, Germany). Primers for endogenous control $\beta$-actin were: 5'-GACCTCTATGCCAACACAGT-3' (forward) and 5'-AGTACTTGCGCTCAGGAGGA-3' (reverse). PCR reaction conditions were: $95^{\circ} \mathrm{C}$ for $45 \mathrm{sec}$, followed by 40 cycles of $95^{\circ} \mathrm{C}$ for $18 \mathrm{sec}$ and $60^{\circ} \mathrm{C}$ for $42 \mathrm{sec}$. Cq values were processed using $2^{-\Delta \Delta C q}$ method (11). Relative expression level of miRNA-21 was normalized to endogenous control $\beta$-actin.

Western blotting. Total protein extraction was performed using cell lysis solutions (Thermo Fisher Scientific, Inc.), and protein concentration was determined by BCA method. Electrophoresis (10\% SDS-PAGE gel) was performed with $30 \mu \mathrm{g}$ of protein per lane. Gel transfer to PVDF membrane was performed under $20 \mathrm{~V}$ for $1 \mathrm{~h}$. Membranes were blocked with 5\% skimmed milk, followed by incubation with primary antibodies including rabbit anti-p-PI3K antibody (1:2,000, ab31392; Abcam, Cambridge, UK), anti-transforming growth factor (TGF)- $\beta 1$ (1:2,000, ab92486; Abcam) anti-GAPDH primary antibody $\left(1: 1,000\right.$, ab37168; Abcam) overnight at $4^{\circ} \mathrm{C}$. The next day, membranes were washed for three times and further incubated with anti-rabbit IgG-HRP secondary antibody (1:1,000, MBS435036; MyBioSource, San Diego, CA, USA) at room temperature for $3 \mathrm{~h}$. After washing, ECL (Sigma-Aldrich; Merck KGaA) method was used to detect signals. Images were processed using Image $\mathbf{J}$ software to calculate the relative expression level of phosphatase and tensin homolog (PTEN) and TGF- $\beta 1$ according to endogenous control GAPDH.

Statistical analysis. The data was presented as the mean \pm standard deviation. SPSS v. 19.0 (SPSS, Inc., Chicago, IL, USA) was used for all statistical analyses. Measurement data were expressed by mean \pm standard deviation, and comparisons between two groups were performed using an unpaired t-test, and comparisons among multiple groups were performed by one-way analysis of variance and the LSD post hoc test. Gender was compared using a Chi-square test. $\mathrm{P}<0.05$ was considered to indicate a statistically significant difference.

\section{Results}

Comparison of expression levels of miRNA-21 in tumor and adjacent healthy tissues of osteosarcoma patients. Expression of miRNA-21 in tumor tissues and adjacent healthy tissues of osteosarcoma patients was detected by RT-qPCR. As shown in Fig. 1, although differential expression levels of miRNA-21 in tumor tissues or adjacent healthy tissues were found among patients, expression levels of miRNA-21 were significantly higher in tumor tissues than those in adjacent healthy tissues in 45 out of 46 patients. Those data suggest that miRNA-21 overexpression is very likely involved in the pathogenesis of osteosarcoma.

Serum levels of miRNA-21 increased with the increased $T$ stage of osteosarcoma. Patients with osteosarcoma $(n=46)$ were divided into $\mathrm{T} 1, \mathrm{~T} 2$ and $\mathrm{T} 3$ groups according to $\mathrm{T}$ staging. Compared with healthy controls, serum levels of miRNA-21 were significantly increased in patients with different $\mathrm{T}$ stages $(\mathrm{P}<0.05$ or $\mathrm{P}<0.01$; Fig. 2). In addition, serum levels of miRNA-21 were also increased with the increased T stage. 


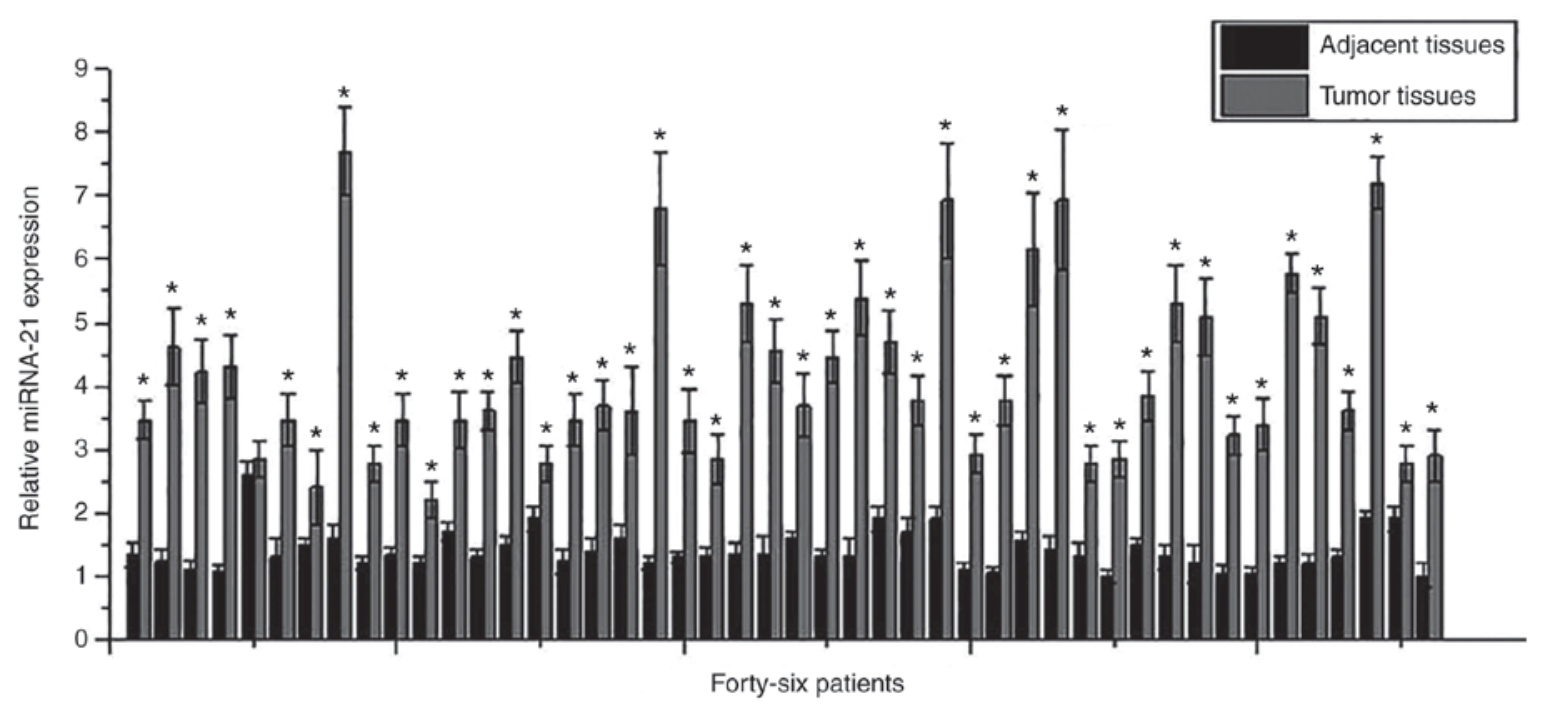

Figure 1. Comparison of miRNA-21 expression in tumor tissues and the adjacent healthy tissues in patients with osteosarcoma. miRNA-21 expression was significantly increased in tumor tissues compared with the adjacent healthy tissues in the majority of patients. "P<0.05 vs. the adjacent healthy tissue. miRNA, microRNA.

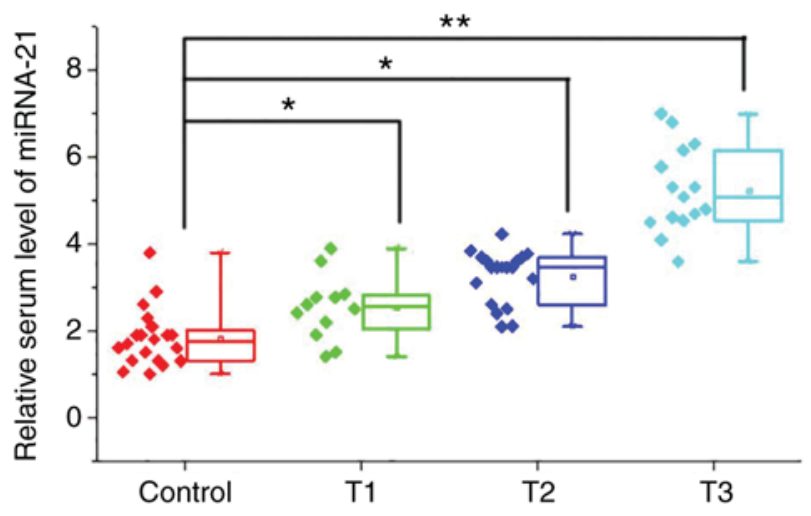

Figure 2. Serum levels of miRNA-21 increase as the T stage of osteosarcoma increases. Compared with the healthy controls, the serum levels of miRNA-21 were significantly increased in patients with different $\mathrm{T}$ stages. In addition, the serum levels of miRNA-21 also increased as the T stage increased. ${ }^{*} \mathrm{P}<0.05$ and ${ }^{* *} \mathrm{P}<0.01$ vs. the adjacent healthy tissue. miRNA, microRNA.

Diagnostic values of serum levels of miRNA-21 for patients with different stages of osteosarcoma. ROC curve analysis was performed to evaluate the diagnostic values of serum levels of miRNA-21 for patients with different stages of osteosarcoma (Fig. 3). The area under the curve (AUC) of serum levels of miRNA-21 in the diagnosis of T1 osteosarcoma was 0.7750 with 95\% confident interval of 0.6037 to 0.9463 ( $\mathrm{P}=0.01023)$, AUC for T2 osteosarcoma was 0.9224 with $95 \%$ confident interval of 0.8276 to 1.017 ( $\mathrm{P}<0.0001)$, and AUC for T3 osteosarcoma was 0.9967 with $95 \%$ confident interval of 0.9856 to $1.008(\mathrm{P}<0.0001)$. Those data suggest that serum levels of miRNA-21 can be used to accurately diagnose osteosarcoma, especially cases in advanced stages.

Prognostic values of serum levels of miRNA-21 for patients with osteosarcoma. According to the median serum level of miRNA-21, patients were divided into high level and low level groups. Survival curves of those two groups were plotted using Kaplan-Meier method and were compared by log rank t-test to evaluate the prognostic value of serum miRNA-21 for osteosarcoma. As shown in Fig. 4, overall survival rate of osteosarcoma patient with high serum level of miRNA-21 was significantly lower than that of patients with low serum level of miRNA-21 $(\mathrm{P}<0.05)$.

miRNA-21 knockdown inhibited proliferation of osteosarcoma. CCK-8 assay was performed to measure cell proliferation abilities of two osteosarcoma cell lines MG-63 and U2OS. As shown in Fig. 5, compared with control cells (cells without transfection) and negative control cells (empty plasmid transfection), proliferation rate of osteosarcoma cells was significantly decreased after the miRNA-21 knockdown.

miRNA-21 knockdown promoted PTEN and TGF- $\beta 1$ expression in osteosarcoma cells. It is known that miRNA-21 can target PTEN to achieve its biological function in glioblastoma (12). In addition, miRNA-21 can also regulate TGF- $\beta 1$ pathway to negatively regulate Treg cells (13). PTEN and TGF- $\beta 1$ expression have been proved to participate in proliferation of certain types of tumor cells $(14,15)$. In this study, miRNA-21 knockdown significantly promoted the expression of PTEN and TGF- $\beta 1$ in both of osteosarcoma cell lines (Fig. 6). PTEN is considered to be a tumor suppressor gene (12) and TGF- $\beta 1$ has anti-proliferative activity. Those data indicate that inhibition of miRNA-21 can inhibit osteosarcoma cell proliferation by reducing the expression level of PTEN and TGF- $\beta 1$.

TGF- $\beta 1$ inhibitor treatment reduced the inhibitory effects of miRNA-21 knockdown on osteosarcoma cell proliferation. Our data have shown that miRNA-21 knockdown-mediated increased expression level of TGF- $\beta 1$ is related with the reduced proliferation rate of osteosarcoma cells. Therefore, TGF- $\beta 1$ inhibitor SB431542 (10 nM) was used to treat cells of two osteosarcoma cell lines. As shown in Fig. 7, proliferation rate of osteosarcoma cells was lower than that of control cells but higher than that of cells with SB431542 treatment 

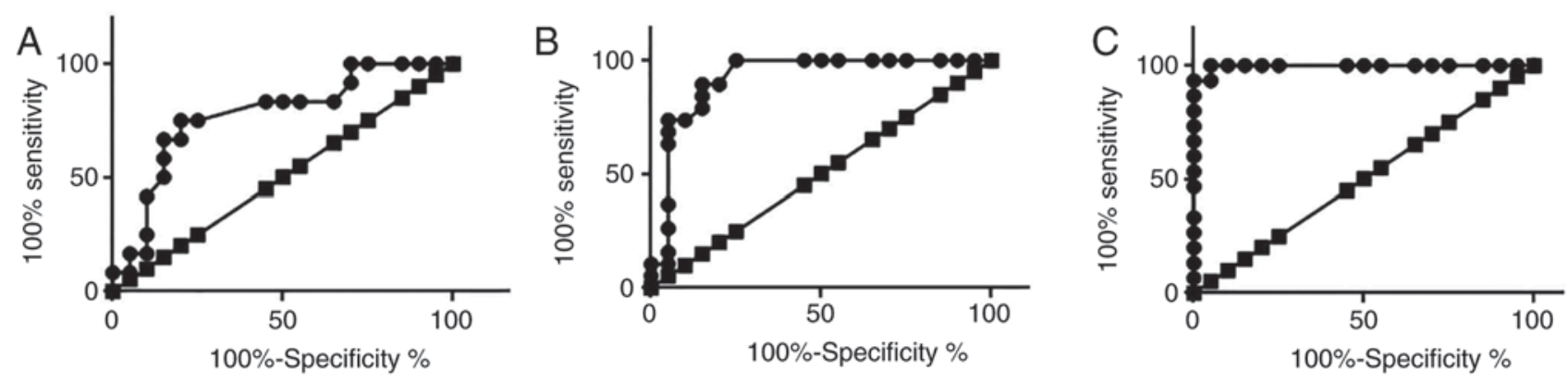

Figure 3. Diagnostic value of serum miRNA-21 for patients with different stages of osteosarcoma. ROC curve analysis of the diagnostic value of serum levels of miRNA-21 for (A) T1, (B) T2 and (C) T3 stage osteosarcoma. The lines with solid circles are the diagnostic curve and the lines with solid squares are the line of identify. Serum levels of miRNA-21 may be used to accurately diagnose osteosarcoma, particularly in cases at advanced stages. miRNA, microRNA.

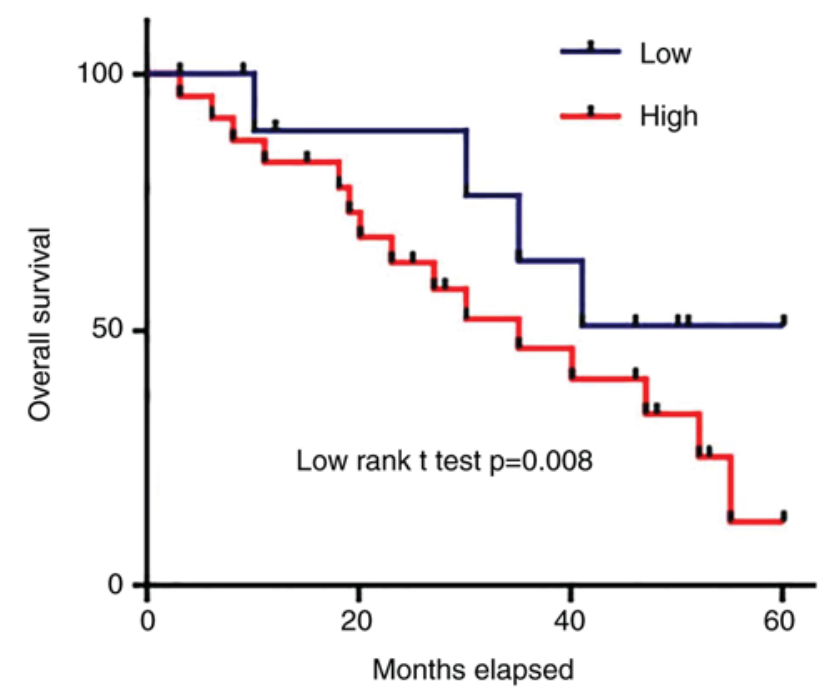

Figure 4. Comparison of survival curves for high and low level miRNA-21 groups. Overall survival rate of patients with osteosarcoma with high serum miRNA-21 was significantly lower compared with patients with low serum miRNA-21. miRNA, microRNA.

and miRNA-21 knockdown. Those data suggest that TGF- $\beta 1$ inhibitor treatment can reduce the inhibitory effects of miRNA-21 knockdown on osteosarcoma cell proliferation.

\section{Discussion}

miRNAs have been proved to play critical roles in the pathogenesis of osteosarcoma (16). Expression of microRNA-199a-3p was downregulated in osteosarcoma tissues comparing with adjacent healthy tissues, and the increased expression level of microRNA-199a-3p is responsible for increased proliferation and migration rates of cancer cells (17). miRNA-218 is also downregulated in osteosarcoma to play a role as tumor suppressor by inhibiting tumor cell migration and invasion (18). miRNA-21 is usually highly expressed in various types of cancer to play its oncogenic functions $(19,20)$. In our study, expression levels of miRNA-21 were significantly higher in tumor tissues than those in adjacent healthy tissues in 45 out of 46 patients. In addition, serum levels of miRNA-21 were also significantly higher in osteosarcoma patients than those in normal controls. Besides that, serum levels of miRNA-21 were increased with the increased $\mathrm{T}$ stage of osteosarcoma.
All those results suggestion that upregulation of miRNA-21 expression is involved in the development of osteosarcoma. miRNA-21 plays its oncogenic functions at least partially by promoting the proliferation of cancer cells, so as to accelerate the growth of tumors (21). In this study, inhibition of miRNA-21 expression significantly promoted the proliferation of two osteosarcoma cell lines, indicating that inhibition of miRNA-21 expression may serve as a potential target for the clinical treatment of osteosarcoma.

Development of pathological processes is usually accompanied by changes of certain substances in blood, and the detection of those substances may provide useful information for diagnosis and prognosis of certain diseases (22). Abnormal expression of miRNA-21 has been proved to serve as promising diagnostic biomarkers for certain types of cancers such as cervical cancer (23). In our study, ROC curve analysis showed that serum levels of miRNA-21 can be used to effectively diagnose osteosarcoma, and the diagnostic value was increased with the increased $\mathrm{T}$ stage of osteosarcoma. Increased expression level of miRNA-21 has been proved to be closely correlated with poor prognosis of some types of cancers such as glioma (24). In our study, survival curves of those two groups were plotted using Kaplan-Meier method and compared by log rank $t$ test to evaluate the prognostic value of serum miRNA-21 for osteosarcoma. We found that the overall survival rate of osteosarcoma patients with high serum level of miRNA-21 was significantly lower than that of patients with low serum level of miRNA-21. Those data suggest that serum miRNA-21 may serve as a promising prognostic and diagnostic biomarker for osteosarcoma.

In the study of triple-negative breast cancer, miRNA-21 was proved to promote the proliferation of cancer cells by targeting PTEN to reduce its expression level (21). TGF- $\beta 1$ plays different roles in different aspects of the cancer pathogenesis. On one hand, TGF- $\beta 1$ reduces cell proliferation rate by inhibiting cell cycle. On the other hand, TGF- $\beta 1$ promotes cell migration and invasion (15). In this study, miRNA-21 knockdown significantly promoted the expression of PTEN and TGF- $\beta 1$ in both of the osteosarcoma cell lines. In addition, TGF- $\beta 1$ inhibitor treatment significantly reduced the inhibitory effects of miRNA-21 knockdown on osteosarcoma cell proliferation. Those data indicate that inhibition of miRNA-21 can inhibit osteosarcoma cell proliferation by reducing the expression level of PTEN and TGF- $\beta 1$. We tried to identify the target site of miRNA-21 on TGF- $\beta 1$, but no promising 

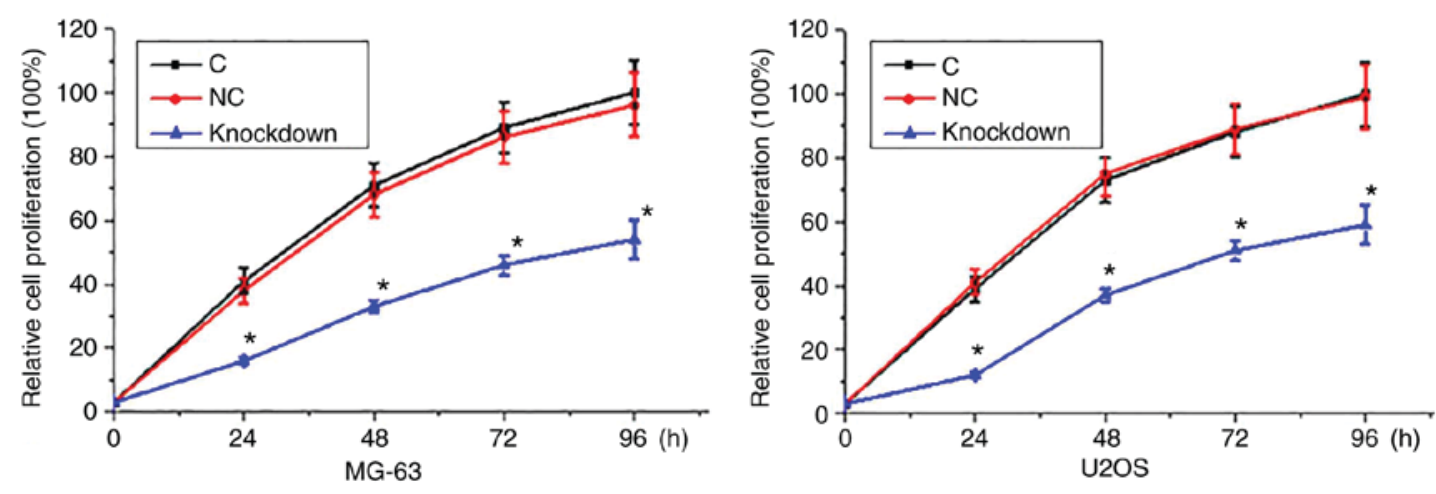

Figure 5. miRNA-21 knockdown inhibits proliferation in osteosarcoma cells. Compared with the control and NC cells, the proliferation rate of osteosarcoma cells was significantly decreased following miRNA-21 knockdown. " $\mathrm{P}<0.05$ vs. the control cells. C, control; NC, negative control; miRNA, microRNA.
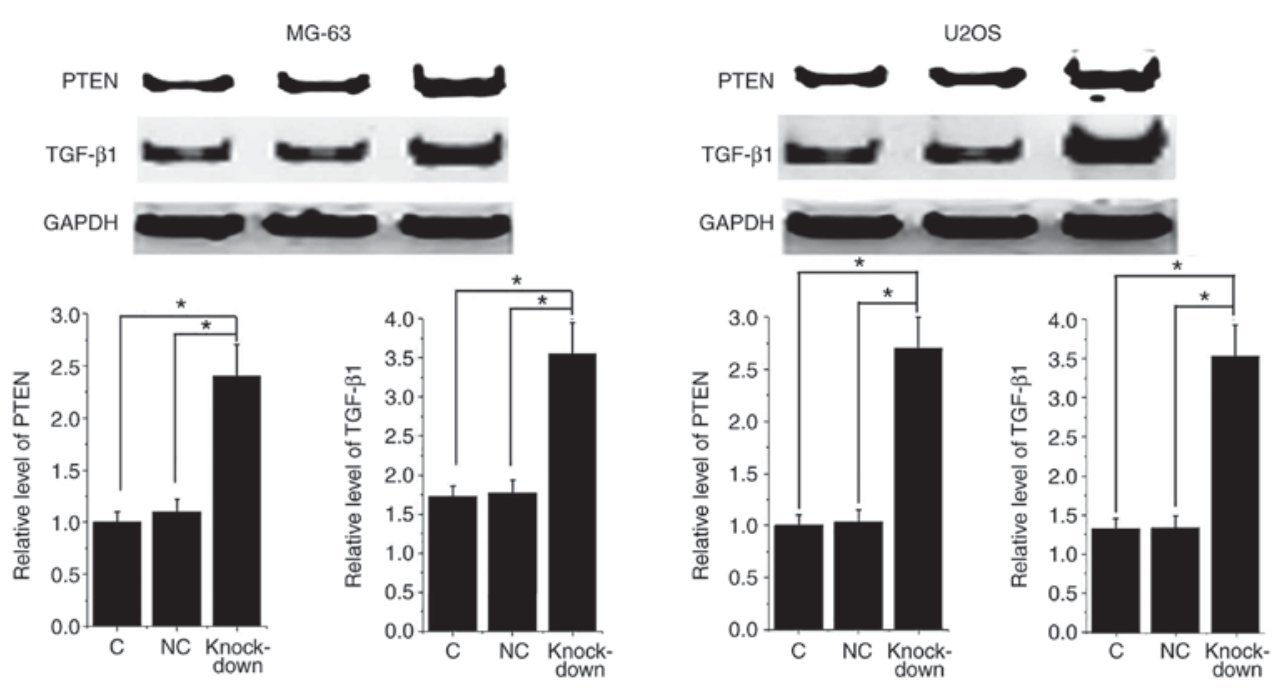

Figure 6. miRNA-21 knockdown promotes PTEN and TGF- $\beta 1$ expression in osteosarcoma cells. miRNA-21 knockdown significantly promoted the protein expression of PTEN and TGF- $\beta 1$ in each of the osteosarcoma cell lines. "P<0.05, as indicated. NC, negative control; PTEN, phosphatase and tensin homolog; TGF, transforming growth factor; miRNA, microRNA.
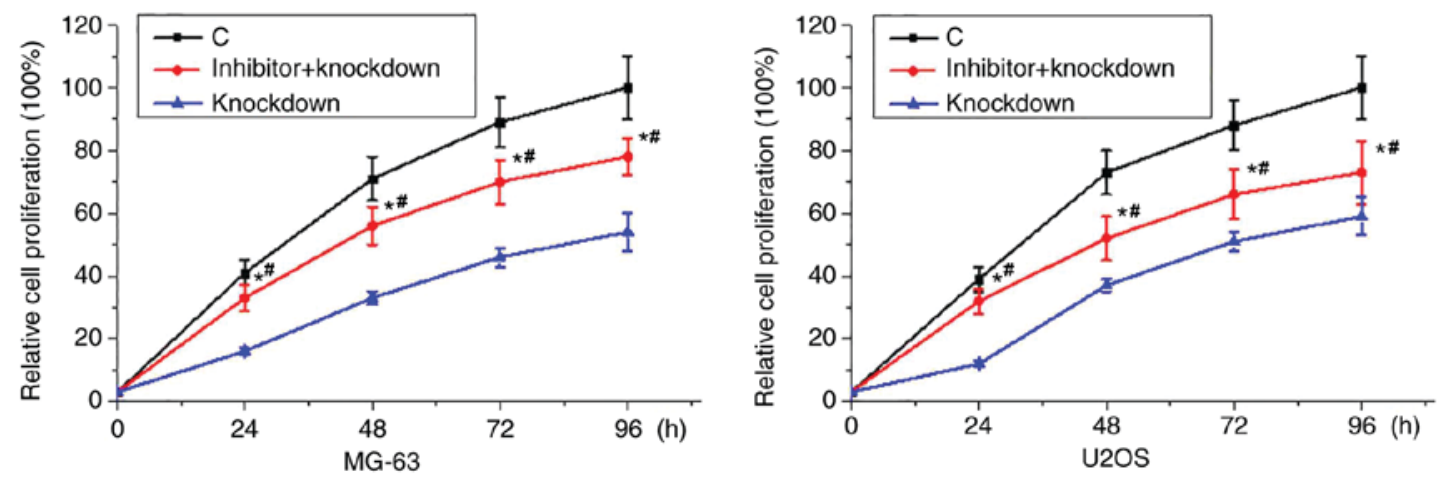

Figure 7. TGF- $\beta 1$ inhibitor treatment reduces the inhibitory effects of miRNA-21 knockdown on osteosarcoma cell proliferation. The proliferation rate of osteosarcoma cells was reduced compared with the control cells and increased compared with the SB431542 treated and miRNA-21 knockdown cells. ${ }^{*} \mathrm{P}<0.05$ vs. the control cells; ${ }^{*} \mathrm{P}<0.05$ vs. the miRNA-21 knockdown cells. TGF, transforming growth factor; miRNA, microRNA.

target sequence was found. Therefore miRNA-21 may target the upstream regulator of TGF- $\beta 1$ to indirectly regulate its expression.

It is worth to note that the application of miRNA-21 as a prognostic marker for osteosarcoma has been evaluated be a previous study (25). Several other studies also reported the involvement of miRNA-21 in osteosarcoma $(20,26)$. However, in those studies the mechanism of the action of miRNA-21 in osteosarcoma has not been investigated. Our study reported the mechanism of the function of miRNA-21 in this disease.

In conclusion, expression levels of miRNA-21 were significantly higher in tumor tissues than those in adjacent 
healthy tissues of most osteosarcoma patients. Serum levels of miRNA-21 were increased with the increased $\mathrm{T}$ stage of osteosarcoma. Serum miRNA-21 can be used to effectively diagnose osteosarcoma and predict the prognosis of the disease. miRNA-21 knockdown inhibited proliferation of osteosarcoma and promoted the expression of PTEN and TGF- $\beta 1$ proteins in those cells, while TGF- $\beta 1$ inhibitor treatment reduced the inhibitory effects of miRNA-21 knockdown on osteosarcoma cell proliferation. Therefore, we conclude that miRNA-21 expression inhibition can inhibit osteosarcoma cell proliferation by targeting PTEN and regulating TGF- $\beta 1$ pathway. However, osteosarcoma is a rare type of cancer, leading to the small sample size in this study. Future studies with bigger sample size are still needed to further confirm the conclusions in this study.

\section{Acknowledgements}

Not applicable.

\section{Funding}

No funding was received.

\section{Availability of data and materials}

The datasets used and/or analyzed during the present study are available from the corresponding author on reasonable request.

\section{Authors' contributions}

$\mathrm{XH}$ and $\mathrm{YZ}$ designed the experiments. XH, LL and YL performed the experiments. XY, HC and QY analyzed the data. YZ wrote the manuscript. All authors have read and approved the final submitted manuscript.

\section{Ethics approval and consent to participate}

The Ethics Committee of Guangzhou General Hospital of Guangzhou Military Command approved the study and all patients provided written informed consent prior to their inclusion.

\section{Patient consent for publication}

Not applicable.

\section{Competing interests}

The authors declare that they have no competing interests.

\section{References}

1. Luetke A, Meyers PA, Lewis I and Juergens H: Osteosarcoma treatment-where do we stand? A state of the art review. Cancer Treat Rev 40: 523-532, 2014.

2. Anderson ME: Update on survival in osteosarcoma. Orthop Clin North Am 47: 283-292, 2016.

3. Botter SM, Neri D and Fuchs B: Recent advances in osteosarcoma. Curr Opin Pharmacol 16: 15-23, 2014.

4. Lin PP and Patel S (eds): Osteosarcoma. In: Bone sarcoma. Springer, New York, NY, pp75-97, 2013.

5. Tsukahara $\mathrm{T}$ and Wada T: Immunotherapy for osteosarcoma. In: Osteosarcoma. Springer, Tokyo, pp31-41, 2016
6. Farfalli GL, Albergo JI, Lobos PA, Smith DE, Streitenberger PD, Pallotta Rodríguez MG and Aponte-Tinao LA: Osteosarcoma lung metastases. Survival after chemotherapy and surgery. Medicina (B Aires) 75: 87-90, 2015 (In Spanish).

7. Ambros V: The functions of animal microRNAs. Nature 431: 350-355, 2004.

8. Bartel DP: MicroRNAs: Genomics, biogenesis, mechanism, and function. Cell 116: 281-297, 2004.

9. Zimmerman AL and Wu S: MicroRNAs, cancer and cancer stem cells. Cancer Lett 300: 10-19, 2011.

10. Shenouda SK and Alahari SK: MicroRNA function in cancer: Oncogene or a tumor suppressor. Cancer Metastasis Rev 28: 369-378, 2009.

11. Livak KJ and Schmittgen TD: Analysis of relative gene expression data using real-time quantitative PCR and the 2(-Delta Delta C(T)) method. Methods 25: 402-408, 2001.

12. Zhou X, Ren Y, Moore L, Mei M, You Y, Xu P, Wang B, Wang G, Jia Z, Pu P, et al: Downregulation of miR-21 inhibits EGFR pathway and suppresses the growth of human glioblastoma cells independent of PTEN status. Lab Invest 90: 144-155, 2010.

13. Li S, Fan Q, He S, Tang T, Liao Y and Xie J: MicroRNA-21 negatively regulates Treg cells through a TGF- $\beta 1 /$ Smad-independent pathway in patients with coronary heart disease. Cell Physiol Biochem 37: 866-878, 2015.

14. Li X, Xie W, Xie C, Huang C, Zhu J, Liang Z, Deng F, Zhu M, Zhu W,Wu R, et al: Curcumin modulates miR-19/PTEN/AKT/p53 axis to suppress bisphenol A-induced MCF-7 breast cancer cell proliferation. Phytother Res 28: 1553-1560, 2014.

15. Li J, Ballim D, Rodriguez M, Cui R, Goding CR, Teng H and Prince S: The anti-proliferative function of the TGF- $\beta 1$ signaling pathway involves the repression of the oncogenic TBX2 by its homologue TBX3. J Biol Chem 289: 35633-35643, 2014.

16. Jones KB, Salah Z, Del Mare S, Galasso M, Gaudio E, Nuovo GJ, Lovat F, LeBlanc K, Palatini J, Randall RL, et al: miRNA signatures associate with pathogenesis and progression of osteosarcoma. Cancer Res 72: 1865-1877, 2012.

17. Duan Z, Choy E, Harmon D, Liu X, Susa M, Mankin H and Hornicek F: MicroRNA-199a-3p is downregulated in human osteosarcoma and regulates cell proliferation and migration. Mol Cancer Ther 10: 1337-1345, 2011.

18. Jin J, Cai L, Liu ZM and Zhou XS: miRNA-218 inhibits osteosarcoma cell migration and invasion by down-regulating of TIAM1, MMP2 and MMP9. Asian Pac J Cancer Prev 14: 3681-3684, 2013.

19. Zhou Y, Huang Z, Wu S, Zang X, Liu M and Shi J: miR-33a is up-regulated in chemoresistant osteosarcoma and promotes osteosarcoma cell resistance to cisplatin by down-regulating TWIST. J Exp Clin Cancer Res 33: 12, 2014.

20. Vanas V, Haigl B, Stockhammer V and Sutterlüty-Fall H: MicroRNA-21 increases proliferation and cisplatin sensitivity of osteosarcoma-derived cells. PLoS One 11: e0161023, 2016.

21. Fang H, Xie J, Zhang M, Zhao Z, Wan Y and Yao Y: miRNA-21 promotes proliferation and invasion of triple-negative breast cancer cells through targeting PTEN. Am J Transl Res 9: 953-961, 2017.

22. Jacobson S, Kechris K, Sun W, Yang J, Chen TH, Barr RG, Basta P, Bleecker ER, Couper D, Curtis JF, et al: Blood biomarker quantitative trail loci in chronic obstructive pulmonary disease. B93. Genetic signatures of Asthma: Key to endotypes? Am Thorac Soc 191: A3627, 2015.

23. Han Y, Xu GX, Lu H, Yu DH, Ren Y, Wang L, Huang XH, Hou WJ, Wei ZH, Chen YP, et al: Dysregulation of miRNA-21 and their potential as biomarkers for the diagnosis of cervical cancer. Int J Clin Exp Pathol 8: 7131-7139, 2015.

24. Shi R, Wang PY, Li XY, Chen JX, Li Y, Zhang XZ, Zhang CG, Jiang T, Li WB, Ding W and Cheng SJ: Exosomal levels of miRNA-21 from cerebrospinal fluids associated with poor prognosis and tumor recurrence of glioma patients. Oncotarget 6: 26971-26981, 2015.

25. Yuan J,Chen L,Chen X, Sun W and Zhou X: Identification of serum microRNA-21 as a biomarker for chemosensitivity and prognosis in human osteosarcoma. J Int Med Res 40: 2090-2097, 2012.

26. Ziyan W, Shuhua Y, Xiufang W and Xiaoyun L: MicroRNA-21 is involved in osteosarcoma cell invasion and migration. Med Oncol 28: 1469-1474, 2011.

This work is licensed under a Creative Commons Attribution-NonCommercial-NoDerivatives 4.0 International (CC BY-NC-ND 4.0) License. 